\title{
From Bistability to Coupling-Induced Oscillations in a Two-Habitat Model for the Rotifer Population Dynamics
}

\author{
A.B. Medvinsky ${ }^{a 1}$, M.M. Gonik ${ }^{a}$, A.V. Rusakov ${ }^{a}$, H. Malchow ${ }^{b}$ \\ ${ }^{a}$ Institute of Theoretical and Experimental Biophysics, \\ Pushchino, Moscow Region, 142290, Russia \\ ${ }^{b}$ Institute of Environmental Systems Research, \\ University of Osnabrück, D-49069 Osnabrück, Germany
}

\begin{abstract}
We study the role of interactions between habitats in rotifer dynamics. For this purpose we use a modified version of the Consensus model. The Consensus model has been shown to be realistic enough to reproduce distinguishing features of the rotifer species dynamics. Being uncoupled, intrinsically bistable rotifer populations, which inhabit the regions under different environmental conditions, do not impact each other. We show that migration of the rotifers between the habitats leads to the transformation of bistability to oscillatory dynamics. The coupling-induced oscillations of the rotifer biomass are shown to be either regular or irregular depending on the value of the model parameters, which describe environmental conditions and the biomass exchange between the habitats. We show that the irregular oscillations are characterized by positive values of the Lyapunov exponent, which means that they are chaotic.
\end{abstract}

Keywords: bistability, coupling-induced oscillations, chaos, rotifer population dynamics AMS subject classification: 92D25

\section{Introduction}

The current state of knowledge of plankton dynamics principles is unsatisfactory on empirical as well as theoretical grounds. The main problem is the complexity of plankton dynamics, which are impacted by both the inherent non-linearity underlying changes in plankton abundance in a habitat and some external factors. Among the many external factors, the exchange of plankton

\footnotetext{
${ }^{1}$ Corresponding author. E-mail: medvinsky @iteb.ru
} 
biomass between habitats, which can lead to the emergence of new dynamical regimes, occupy a highly important place. As a result, plankton abundance has been shown to undergo occasional transitions from regular to chaotic dynamics and vice versa $[1,2]$.

It is remarkable that some characteristic features of complex plankton dynamics can be simulated with the use of very simple conceptual mathematical models $[2,3,4,5,6,7,8,9,10,11]$. Within the framework of such an approach Snell and Serra [12] have developed a disctrete-time model (Consensus model) of population dynamics of rotifers. Rotifers are planktonic animals. The name "rotifer" is derived from the Latin word meaning "wheel-bearer". This makes reference to the crown of cilia, whose rapid movement makes rotifers appear to whirl like a wheel. Rotifers inhabit freshwater environments, such as lakes and rivers [13].

It is remarkable that the Consensus model [12] is realistic enough to capture the relevant biology but is simplified to a level where the parameterization of the model becomes possible. Berezovskaya et al. [14] carried out a sophysticated mathematical analysis of the Consensus model and identified a number of the dynamical regimes, including stable equilibrium, periodic oscillations and chaotic oscillations, which correspond closely to the data obtained in the course of field observations. Recently, leaning upon the results obtained by Berezovskaya et al. [14] we have modified the Consensus model in order to study the role of interhabitat exhange of biomass in the dynamics of the rotifer populations in a heterogeneous environment [15]. In particular, we have shown that the biomass exchange between the rotifer populations with different intrinsic dynamical regimes can lead to a spatially confinement of chaos invasion and to distortion of spatial borders of the clusters of synchronized rotifer oscillations [16].

An open question still is what the result of biomass exchange between rotifer populations with not different but identical intrinsic dynamics can be? In this paper, using the model proposed by Snell and Serra [12] and some results of the analysis of the model carried out by Berezovskaya et al. [14], we show that the rotifer populations, that are both bistable when uncoupled, can undergo oscillations in their size resulting from the exchange of biomass between the populations.

\section{The model}

The Consensus model takes the form:

$$
N(t+1)=N(t) \exp \left(-\alpha+\frac{b}{N(t)}-\frac{c}{N^{2}(t)}+\epsilon(t)\right)
$$

where $N(t)$ is the population density at time $t$ with the time unit, which crudely correspounds to one day [12], $\alpha, b$ and $c$ are constants, and $\epsilon(t)$ is a random variable representing exogeneous population density-independent effects on the rotifer population under study. Throughout this paper we exclude the random component of population dynamics from consideration, i.e. $\epsilon(t)=0$. This makes it possible to focus on the inherent features of the dynamics.

The model (2.1) can be simplified by scaling $N \rightarrow b N$. After introducing $\gamma=c / b^{2}$, Eq. (2.1) becomes 


$$
N(t+1)=N(t) \exp \left(-\alpha+\frac{1}{N(t)}-\frac{\gamma}{N^{2}(t)}\right) .
$$

The parameter $\alpha$ in (2.1) and (2.2) characterizes environmental conditions. This parameter is changed as the environmental conditions vary in time or/and in space. The parameter $\gamma$ is species specific [14]. For example, $\gamma=0.057$ for Brachionus rotundiformis, $\gamma=0.076$ for Brachionus lyratus, and $\gamma=0.152$ for Asplanchna girodi $[12,14]$.

The modified Consensus model, which sumulates the dynamics of two coupled habitats populated with the same rotifer species under different environmental conditions, is:

$$
\begin{aligned}
& N_{1}(t+1)=N_{1}(t) \exp \left(-\alpha_{1}+\frac{1}{N_{1}(t)}-\frac{\gamma}{N_{1}^{2}(t)}\right)-k\left(N_{1}(t)-N_{2}(t)\right), \\
& N_{2}(t+1)=N_{2}(t) \exp \left(-\alpha_{2}+\frac{1}{N_{2}(t)}-\frac{\gamma}{N_{2}^{2}(t)}\right)-k\left(N_{2}(t)-N_{1}(t)\right),
\end{aligned}
$$

where $N_{1}(t)$ and $N_{2}(t)$ are the densities of the rotifer populations in the first and second habitats, respectively, and $k$ is the coupling coefficient.

Model (2.3) - (2.4) implies that reproduction and dispersal of planktoners occur simultaneously (with a precision of one time step; see [17] for more details). Considering that both $N_{1}(t)$ and $N_{2}(t)$ are not negative, the value of the coupling coefficient $k$ has to be less than a critical value, which is species-specific and also depends on the parameters $\alpha_{1}$ and $\alpha_{2}$ [15]. We consider two habitats under different environmental conditions: $\alpha_{1} \neq \alpha_{2}$.

\section{Results}

Figure 1 shows $N_{t+1}$ plotted as a function of $N_{t}$ for parameter values $[12,14]$ that represent the inherent dynamics of a rotifer population. One can see that the population is bistable. The dynamics of the population is characterized by three equilibria, two of them stable. The basins of attraction of the stable equlibria are separated by the unstable equilibrium (Figure 1). In other words, the size of the population goes to a constant value. The constant value is zero if the initial population size is less than a critical size, otherwise it is nonzero.

Such type of population dynamics (the so-called Allee effect [18]) has been found for a number of rotifer species. All the species are situated at the same domain of the Consensus model parameter space (domain II in Figure 2); different domains correspond to different types of the population dynamics (see [14] for more detail). As an example, positions of three bistable rotifer populations, which belong in domain II, are shown (Figure 2). They are Brachionus rotundiformis, Brachionus lyratus and Asplanchna girodi.

Thus, the dynamics of the rotifer populations can exhibit bistability if the populations are not subjected to external influences resulting, for example, from the heterogeneity, which is typical of aquatic environments [2]. It may be assumed that interhabitat exchange of biomass arising from an environmental heterogeneity, can affect the bistability of the population dynamics. Our simulations 


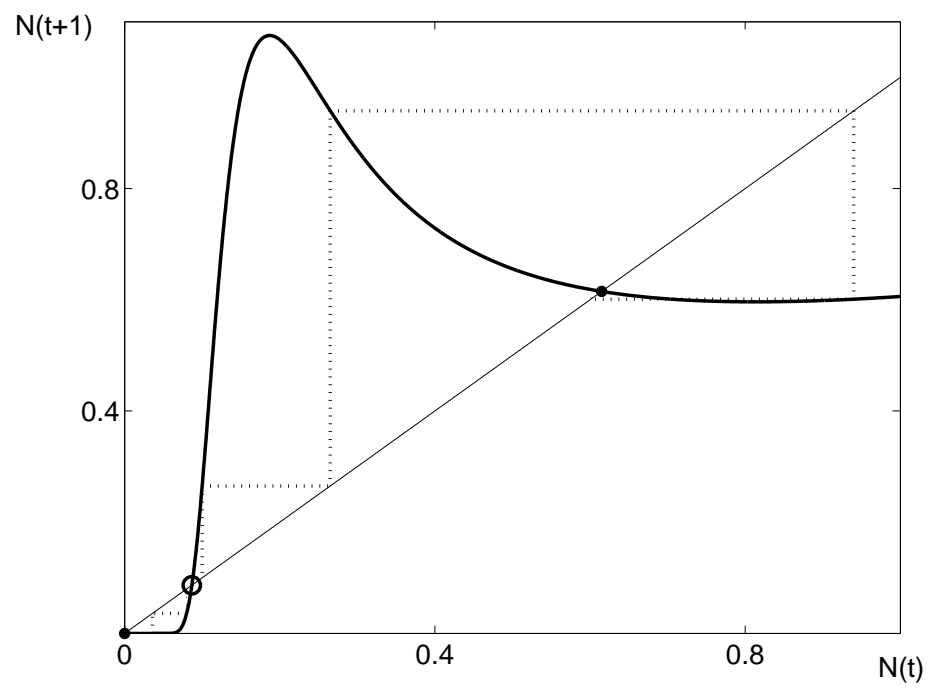

Figure 1: A phase portrait of model (2.2) for model parameters characteristic of Brachionus lyratus: $\alpha=1.425, \gamma=0.076[12,14]$. The black points mark stable equilibria while the circle corresponds to the unstable equilibrium.

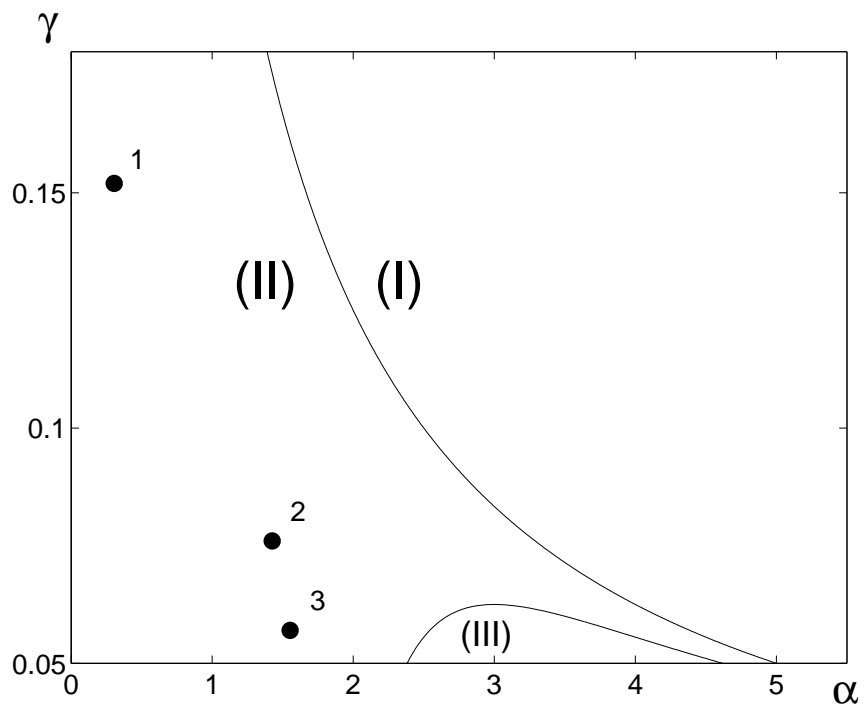

Figure 2: Positions of the rotifer species inside domain II of the parameter space: 1 - Asplanchna girodi, 2 - Brachionus lyratus, 3 - Brachionus rotundiformis. The domains are: I - total extinction, II - bistability, III - zero stable and periodic orbits [14]. 
show that this is the case. An example shown in Figure 3 demonstrates variations in the character of the rotifer (Brachionus lyratus) population dynamics under changes of the value of the coupling coefficient $k$.

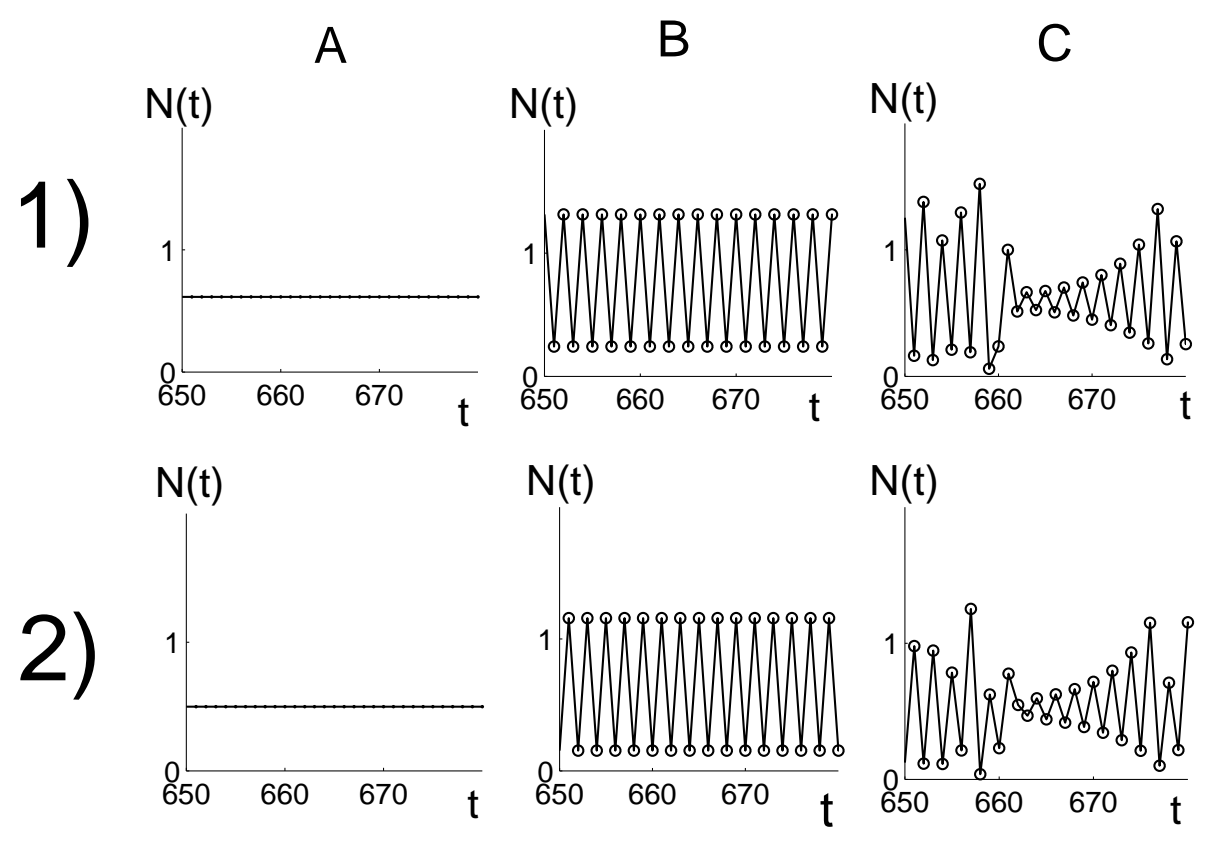

Figure 3: Three types of the rotifer (Brachionus lyratus) population dynamics in each of the two habitats under different values of the coupling coefficient $k: k=0$ (A), $k=0.35$ (B), $k=0.42$ (C); $\alpha_{1}=1.425, \alpha_{2}=1.7 ; \gamma=0.076$ [14].

One can see that being uncoupled (i.e. at $k=0$ ) the rotifer populations are stationary stable (Figure 3A). However, as soon as the value of $k$ is greater than a critical value, which depends on the model parameters $\alpha$ and $\gamma$, the dynamics of the rotifer populations becomes oscillatory. The growth of the overcritical values of the coupling coefficient $k$ is followed by the transition from regular oscillations of the rotifer population density (Figure 3B) to the irregular time series (Figure 3C). Notice that the irregular oscillations are chaotic, which follows from the fact that the Lyapunov exponents of the irregular time series (in contrast to the regular ones) are greater than zero. The Lyapunov exponent is a well-known measure of sensitive dependence. The Lyapunov exponent, if positive, indicates that small differences in initial conditions diverge exponentially over time. This sensitivity to initial conditions is indicative of chaos; see, for example, [19]). To calculate the Lyapunov exponents, we followed the method given in [20]. In our case, the values of the Lyapunov exponents typical of the chaotic regimes fall within the wide range between 0.002 and 0.07 .

The bifurcation diagram (Figure 4) demonstrates the dependence of the population dynamics on the value of the coupling coefficient $k$ in more detail. One can see that increase in the value of $k$ leads to the Hopf bifurcation and subsequent period doubling followed by chaos. The windows of periodic behavior [21, 22], which are embedded in the region of chaos are also seen. 

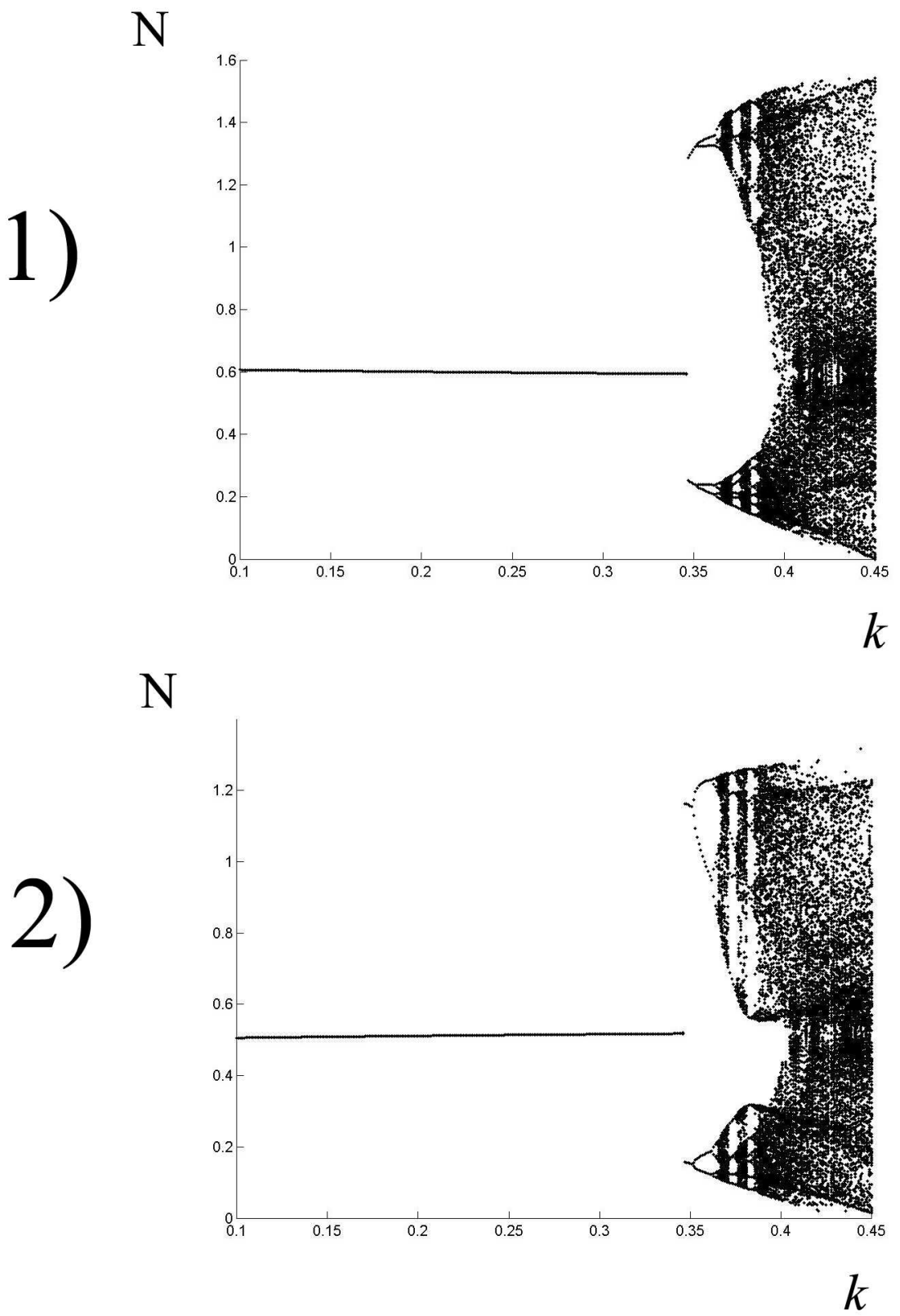

Figure 4: The bifurcation diagrams demonstrating three types of the rotifer (Brachionus lyratus) population dynamics in each of the two habitats under different values of the coupling coefficient $k ; \alpha_{1}=1.425, \alpha_{2}=1.7, \gamma=0.076$. 
A dependence of the rotifer population dynamics on the parameter $\alpha_{2}$, characterizing environmental conditions in the second habitat, is shown in Figure 5. Namely, Figure 5 shows the bifurcation diagrams that characterize the rotifer (Brachionus lyratus) population dynamics in each of the two coupled habitats in relation to the value of the parameter $\alpha_{2}$; the parameter $\alpha_{1}$ is held constant and takes the value given in $[12,14]$, i.e. $\alpha_{1}=1.425$. One can see from Figure 5 that changes of environmental conditions in one of the habitats, that are reflected in the changes of the value of the parameter $\alpha_{2}$, are suitable for leading to the transition from bistability (as in Figure 1) to oscillatory behavior, both regular and irregular. Irregular oscillations are also chaotic here.

Notice that transition from equilibrium to chaos resulting from period doubling takes place under both increase and decrease in the value of the parameter $\alpha_{2}$ (Figure 5). Increase in the value of this parameter leads to the Hopf bifurcation and subsequent period doubling in a very narrow interval of the values of the parameter $\alpha_{2}$ : between 1.4620 and 1. 4739 (not shown). Decrease in the value of the parameter $\alpha_{2}$, contrastingly, leads to period doubling in a wide $\alpha_{2}$ range (Figure $5)$.

In contrast to the Brachionus lyratus population dynamics (Figures 3-5), only regular oscillations followed bistability in the Asplanchna girodi ( $\gamma=0.152$ [14]) coupled populations, while in the Brachionus rotundiformis ( $\gamma=0.057$ [14]) populations the bistability was followed by nothing but chaotic oscillations of the population density as the parameter $\alpha_{2}$ was increased. Thus, the character of the changes in the rotifer population dynamics is species-specific and also depending on both the enviromental conditions and interhabitat biomass exchange.

\section{Concluding remarks}

Bistability is often considered as a common trait of population dynamics [23, 24, 25, 26]. The sophysticated mathematical analysis carried out by Berezovskaya et al. [14] has revealed that the bistable rotifer species, that demonstrate exponentially damped biomass oscillations, fall into a large domain (domain II) of the Consensus model parameter space (Figure 2). However, not only exponentially damped time series but also persistent oscillations of the plankton abundance have been observed in natural aquatic communities [2, 12, 27, 28, 29], suggesting that stable equilibria and oscillatory dynamics may transform to each other.

Interestingly, transformation of bistability into oscillatory kinetics, which results from coupling between similar elements, has been found in some physico-chemical systems, too [30, 31]. This allowed us to think that oscillatory rotifer dynamics can arise from coupling between the populations, which belong to the same domain in the model parameter space.

We found that the biomass exchange between the bistable rotifer populations inhabiting two areas with different environmental conditions (i.e. under $\alpha_{1} \neq \alpha_{2}$ in terms of the Consensus model) can give rise to oscillations of the rotifer abundance. Hence the heterogeneity of the environment can lead to the essential transformation of the rotifer population dynamics. This phenomenon can be common in nature since aquatic environments have been shown to be extremely heterogeneous. In addition to very changeable transient spatial patterns, many more stable structures also exist, 

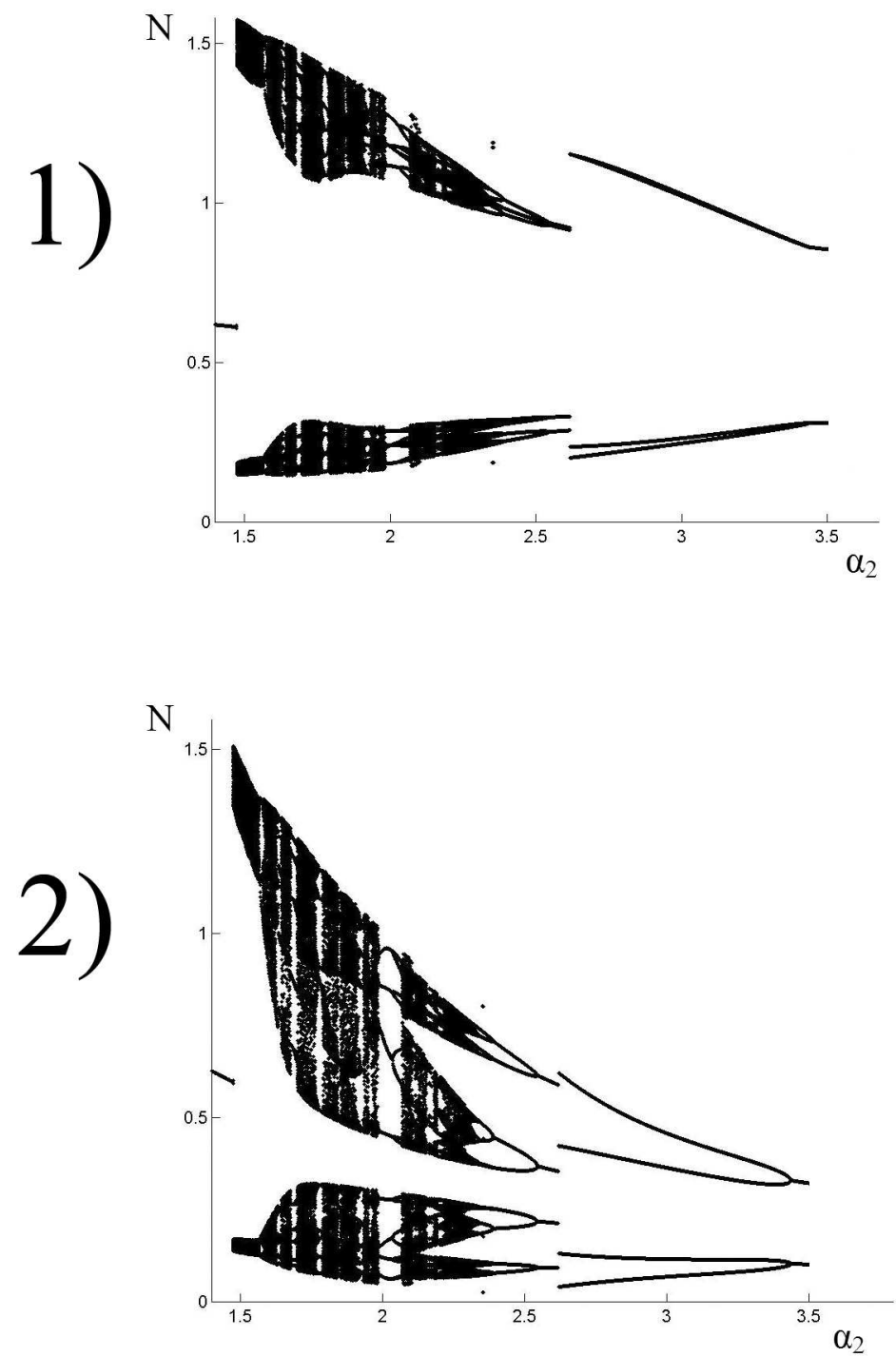

Figure 5: The bifurcation diagrams demonstrating three types of the rotifer (Brachionus lyratus) population dynamics in each of the two habitats under different values of the parameter $\alpha_{2}, \alpha_{1}=$ $1.425, k=0.38$. 
associated with ocean fronts [32, 33], cyclonic rings [34] and so-called meddies [35]. Spatial heterogeneity has also been found in offshore marine areas, lakes and rivers [36, 37, 38, 39, 40].

It is noteworthy that the character of the oscillations of the model rotifer abundance depends essentially on the interhabitat biomass exchange and difference in environmental conditions. In other words, the oscillatory rotifer population dynamics can become chaotic as numerical values of the coupling coefficient $k$ or/and the parameter $\alpha_{2}$ vary (Figures $3-5$ ). In this connection it should be noted that increase in the value of the parameter $\alpha_{2}$ leads to period doubling (followed by chaotic dynamics) in an extremely narrow $\alpha_{2}$ interval. As a result, even small variations in the parameter $\alpha_{2}$ value can lead to sudden "jumps" from equilibrium to chaos and vice versa. Interestingly, immediate transitions from equilibrium to chaotic dynamics have been observed experimentally $[41,42]$.

One can see that the chaotic oscillatory regimes emerge in the neighboring habitats for the same set of the model parameter values (see Figure 5 as an example). In such a way, the couplinginduced chaos resulting from the biomass exchange between the habitats belonging in the same domain of the parameter space (domain II in Figure 2) may expand widely through aquatic ecosystems. Notice that in contrast to the above-mentioned intradomain biomass exchange, coupling between the rotifer populations, which belong to different domains of the Consensus model parameter space, can lead to confinement of the invasion of chaos resulting from invariance of the attractor size under transformation of regular oscillations into chaotic ones [16]. Notice that such a "dynamical" invasion does not imply biological invasion, i.e. the appearance of a foreign invader, since we speculate that $\gamma_{1}=\gamma_{2}$.

Our results show that multihabitat interactions can induce the process of expansion of nonintrinsic types of the rotifer population dynamics, also known as dynamical invasion [16]. The further investigation of the "dynamical" invasion is a challenging problem.

\section{Acknowledgements}

We are gratefull to unknown referees for their comments. This work was partially supported by RFBR and DFG grants.

\section{References}

[1] A.B. Medvinsky, I.A. Tikhonova, R.R. Aliev, B.-L. Li, Z.-S. Lin, H. Malchow. Patchy environment as a factor of complex plankton dynamics. Phys. Rev. E, 64 (2001), 021915 (7 pages).

[2] A.B. Medvinsky, S.V. Petrovskii, I.A. Tikhonova, H. Malchow, B.-L. Li. Spatiotemporal complexity of plankton and fish dynamics. SIAM Rev., 44 (2002), 311-370.

[3] L.A. Segel, J.L. Jackson. Dissipative structure: An explanation and an ecological example. J. Theor. Biol., 37 (1972), 545-559. 
[4] S.A. Levin, L.A. Segel. Hypothesis for origin of planktonic patchiness. Nature, 259 (1976), 659.

[5] M.J.R. Fasham. The statistical and mathematical analysis of plankton patchiness. Oceanography and Marine Biol. Annual Rev., 16 (1978), 43-79.

[6] M.R. Droop. 25 years of algal growth kinetics. Botanica Marina, 43 (1983), 159-183.

[7] S. Rinaldi, S. Muratori. Conditioned chaos in seasonally perturbed predator-prey models. Ecol. Model., 69 (1993), 79-97.

[8] M.J. Behrenfeldt, P.G. Falkowski. A consumer's guide to phytoplankton primary productivity models. Limnology and Oceanography, 42 (1997), 1479-1491.

[9] M. Scheffer. Ecology of Shallow Lakes. Chapman and Hall, London, 1998.

[10] J. Huisman, F.J. Weissing. Biodiversity of plankton by species oscillations and chaos. Nature, 402 (1999), 407-410.

[11] A.B. Medvinsky, D.A. Tikhonov, J. Enderlein, H. Malchow. Fish and plankton interplay determines both plankton spatio-temporal pattern formation and fish school walks. Nonlinear Dynamics, Psychology, and Life Sciences, 4 (2000), 135-152.

[12] T.W. Snell, M. Serra. Dynamics of natural rotifer populations. Hydrobiologia, 368 (1998), $29-35$.

[13] T. Nogrady, R.L. Wallace, T.W. Snell. Rotifera. V. 1. Biology, Ecology and Systematics. SPB Academic Publishing, The Hague, 1993.

[14] F. Berezovskaya, G. Karev, T.W. Snell. Modeling the dynamics of natural rotifer populations: Phase-parametric analysis. Ecol. Compl., 2 (2005), 395-410.

[15] A.B. Medvinsky, M.M. Gonik, F.S. Berezovskaya, B.-L. Li, H. Malchow. Rotifer population dynamics in two coupled habitats: invasion of chaos. Biological Invasions, 7 (2005), 877-883.

[16] M.M. Gonik, A.B. Medvinsky. Confinement of dynamical chaos expansion in rotifer populations inhabiting heterogeneous environments: An effect of attractor size invariance. Nonlinear Dynamics, Psychology, and Life Sciences, 11 (2007), 185-196.

[17] J. Bascompte, R.V. Solé. Appropriare formulations for dispersal in spatially structured models: reply. J. Animal Ecol., 64 (1995), 665-666.

[18] W.C. Allee. Animal Aggregations: A Study In General Sociology. University of Chicago, Chicago, 1931.

[19] D. Kaplan, L. Glass. Understanding Nonlinear Dynamics. Springer, New York, 1995. 
[20] H. Kantz, T. Schreiber. Nonlinear Time Series Analysis. Cambridge University, Cambridge, 1997.

[21] R. May. Biological populations obeying difference equations: stable points, stable cycles and chaos. J. Theor. Biol., 51 (1975), 511-524.

[22] R. May. Simple mathematical models with very complicated dynamics. Nature, 261 (1976), 459-467.

[23] A.D. Armand, M.A. Vedyushkin. Trigger Geosystems. Institute of Geography, Moscow, 1989.

[24] I. Hanski. Metapopulation Ecology. Oxford University, Oxford, 1999.

[25] S.V. Petrovskii, B.-L. Li. Exactly Solvable Models of Biological Invasion. Chapman and Hall/CRC, Boca Raton, 2006

[26] R.V. Solé, J. Bascompte. Self-Organization in Complex Ecosystems. Princeton University, Princeton, 2006.

[27] I. Valiela. Marine Ecological Processes. Springer, New York, 1995.

[28] A.B. Medvinskii, S.V. Petrovskii, I.A. Tikhonova, D.A. Tikhonov, B.-L. Li, E. Venturino, H. Malchow, G.R. Ivanitskii. Spatio-temporal pattern formation, fractals, and chaos in conceptual ecological models as applied to coupled plankton-fish dynamics. Physics-Uspekhi, 45 (2002), 27-57.

[29] H. Holst, H. Zimmermann, H. Kausch, W. Koste. Temporal and spatial dynamics of planktonic rotifers in the Elbe estuary during spring. Estuarine, Coastal and Shelf Science, 47 (1998), 261-273.

[30] V.P. Zhdanov, D. Kulginov, B. Kassemo. Oscillatory kinetics of reactions on solid surfaces resulting from bistability and diffusion limitations. Physical Review E, 53 (1996), R3013R3016.

[31] V. In, A.R. Bulsara, A. Palacios, P. Longhini, A. Kho, J.D. Neff. Coupling-induced oscillations in overdamped bistable systems. Physical Review E, 68 (2003), 045102 (4 pages).

[32] K.N. Fedorov. The Structure of Ocean Fronts. Gidrometeoizdat, Leningrad, 1983.

[33] J.A. Barth. Stability of a coastal upwelling front. 1. Model development and a stability theorem. J. Geophys. Res., 94 (1989), No. C8, 10844-10856.

[34] M.J. Bowman, S.M. Chiswell, P.L. Lapennas, R.A. Murtagh. Coastal upwelling, cyclogenesis, and squid fishing near Cape Farewell, New Zealand. In: H.G. Gade (ed.) Coastal Oceanography, pp. 279-310, New York: Plenum, 1983. 
[35] L. Armi, D. Hebri, N. Oakey, J. Price, P.L. Richardson, T. Rossby, B. Ruddick. The history and decay of a Mediterranean salt lens. Nature, 333 (1988), 649-651.

[36] V.D. Fedorov, T.G. Gilmanov. Ecology. Moscow University, Moscow, 1980.

[37] N.G. Hairston Jr., E.J. Olds. Population differences in the timing of diapause: adaptation in a spatially heterogeneous environment. Oecologia, 61 (1984), 42-48.

[38] S. Czachorowski. Concomitance of caddis fly (Trichoptera) larvae in four Masurian lakes differing trophically (north-eastern Poland). Acta Hydrobiol., 36 (1994), 213-225.

[39] G.G. Matishov, N.V. Druzkov, P.R. Makarevich, E.I. Druzkova, A.A. Namyatov. Identification of habitats in a pelagic zone of Kola Bay (Barents Sea) with the use of the analysis of structure of microplankton communities. Proceedings of the Russian Academy of Sciences, 372 (2000), 568-570.

[40] J.P. Dahlgren, J. Ehrlén. Distribution patterns of vascular plants in lakes - the role of metapopulation dynamics. Ecography, 28 (2005), 49-58.

[41] G.S. Copeland, F.C. Moon. Chaotic flow-induced vibration of a flexible tube with end mass. Journal of Fluids and Structures, 6 (1992), 705-718.

[42] V.A. Il'in, B.L. Smorodin. Periodic and chaotic regimes of liquid dielectric convection in a horizontal capacitor. Technical Physics Letters, 31 (2005), 432-434. 